Supporting information for:

\title{
Effects of Low-level Lipid Peroxidation on the Permeability of Nitroaromatic Molecules across a Membrane. A Computational Study
}

\author{
Hong Yang, ${ }^{+,+, \S}$ Mi Zhou, ${ }^{\ddagger}$, Huarong Li, ${ }^{\star}$ Tong Wei, ${ }^{\star}$ Can Tang, ${ }^{\star}$ Yang Zhou, ${ }^{*},{ }^{*}$ Xinping \\ Long: \\ $\dagger$ School of Materials Science and Engineering, Tsinghua University, Beijing 100084, \\ China. \\ $\$$ Institute of Chemical Materials, Chinese Academy of Engineering and Physics, \\ Mianyang 621900, China;
}

*To whom Correspondence should be addressed: zhouy@caep.cn (Y. Zhou) 


\section{Force field parameters}

The parameters required to describe the oxidized group (aldehyde group, - $\mathrm{CHO}$ ) were derived from quantum chemistry calculation based on $\mathrm{ffTK}^{1}$ and Gaussian $09^{2}$ programs, according to the following protocol:

(1) We first obtained the LJ parameters of aldehyde groups from the CGenFF, the parameters of carbon, oxygen and hydrogen atoms were taken from aldehyde C (CG2O4), aldehyde O (OG2D1) and aldehyde H (HGR52), respectively.

(2) Propanal (Figure S1) was chosen as a model molecules for the calculation of partial charges and bonded parameters for oxidized lipid tails. According to the methods recommended by ffTK program, we calculated the propanal-water interactions using $\mathrm{HF} / 6-31 \mathrm{G}^{*}$ level of theory. The partial charges were obtained by ensuring the reproduction of propanal-water interactions.

(3) Bond and angles parameters were computed by fitting the potential energy surface of target molecule (propanal) as implemented in ffTK, and the MP2/6-31G* level of theory was utilized.

(4) The dihedral parameters were calculated by a holistic approach in which all dihedrals were simultaneously fit to a torsional potential energy surface as employed in the ffTK modul. The MP2/6-31G* level of theory was used.

Some parameters for describing the oxidized phospholipids were directly taken from CHARMM36 force field and CGenFF, and the additional parameters from above-mentioned quantum chemistry calculations are presented in Table S1-S4. 


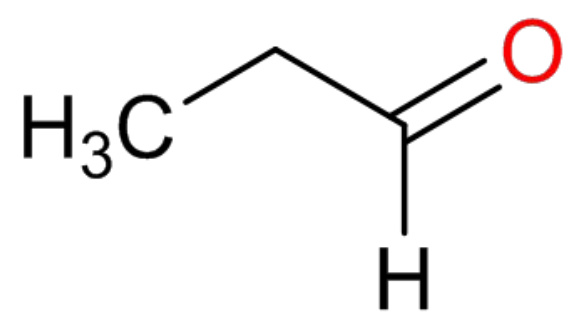

\section{propanal}

Figure. S1 Illustration of the model molecules for the calculation of additional force field parameters.

Table S1. Atomtype definition, charges and connectivity for alOX.

\begin{tabular}{|c|c|c|c|}
\hline$\cdots$ & Atom name & Atom type & charge \\
\hline & $\mathrm{C} 27$ & CTL2 & -0.180 \\
\hline$\longrightarrow \mathrm{H} 7 \mathrm{R}$ & H7R & HAL2 & 0.090 \\
\hline & $\mathrm{H} 7 \mathrm{~S}$ & HAL2 & 0.090 \\
\hline $8 \mathrm{~S}-\mathrm{C} 28-\mathrm{H} 8 \mathrm{R}$ & $\mathrm{C} 28$ & CTL2 & -0.180 \\
\hline & H8R & HAL2 & 0.090 \\
\hline $\mathrm{H} 19-\mathrm{C} 29-\mathrm{H} 92$ & $\mathrm{H} 8 \mathrm{~S}$ & HAL2 & 0.090 \\
\hline & $\mathrm{C} 29$ & CTL2 & -0.180 \\
\hline $\mathrm{C} 210-\mathrm{H} 101$ & H91 & HAL2 & 0.090 \\
\hline & H92 & HAL2 & 0.090 \\
\hline OP1 & $\mathrm{C} 210$ & CG2O4 & 0.425 \\
\hline & H101 & HGR52 & 0.090 \\
\hline & OP1 & OG2D1 & -0.515 \\
\hline
\end{tabular}


Table S2. Additional bond parameters used in the force field for describing the aldehyde groups in the lipid molecules. Units are presented in the brackets.

\begin{tabular}{cccc}
\hline Atom type \#1 & Atom type \#2 & $\mathrm{b}_{0}[\mathrm{~nm}]$ & $\mathrm{K}_{\mathrm{b}}\left[\mathrm{kJ} \cdot \mathrm{mol}^{-1} \cdot \mathrm{nm}^{-2}\right]$ \\
\hline CTL2 & CG2O4 & 0.1510 & 253942.86 \\
\hline
\end{tabular}

Table S3. Additional angle parameters used in the force field for describing the aldehyde groups in the lipid molecules. Units are presented in the brackets.

\begin{tabular}{ccccc}
\hline Atom type \#1 & Atom type \#2 & Atom type \#3 & $\theta_{0}[$ degree $]$ & $\mathrm{K}_{\theta}\left[\mathrm{kJ} \cdot \mathrm{mol}^{-1} \cdot\right.$ degree $\left.^{-2}\right]$ \\
\hline CTL2 & CTL2 & CG2O4 & 107.319 & 592.529712 \\
CTL2 & CG2O4 & HGR52 & 114.774 & 311.66616 \\
CTL2 & CG2O4 & OG2D1 & 125.97 & 738.30864 \\
HAL2 & CTL2 & CG2O4 & 109.415 & 458.641712 \\
\hline
\end{tabular}

Table S4. Additional dihedral parameters used in the force field for describing the aldehyde groups in the lipid molecules. Units are presented in the brackets, $*$ refers to degree, $\neq$ refers to $\mathrm{kJ} \cdot \mathrm{mol}^{-1}$ and $\mathrm{n}$ indicates multiplicity.

\begin{tabular}{ccccccc}
\hline Atom type \#1 & Atom type \#2 & Atom type \#3 & Atom type \#4 & $\phi_{0}{ }^{[*]}$ & $\mathrm{K}_{\phi}^{[\sharp]}$ & $\mathrm{n}$ \\
\hline CTL2 & CTL2 & CG2O4 & HGR52 & 180 & 2.88696 & 2 \\
HAL2 & CTL2 & CG2O4 & HGR52 & 0 & 1.744728 & 3 \\
CTL2 & CTL2 & CG2O4 & OG2D1 & 180 & 32.86532 & 1 \\
HAL2 & CTL2 & CTL2 & HAL2 & 0 & 0.81588 & 3 \\
HAL2 & CTL2 & CG2O4 & OG2D1 & 180 & 25.426168 & 1 \\
HAL2 & CTL2 & CTL2 & CG2O4 & 180 & 7.80316 & 1 \\
\hline
\end{tabular}


(a)

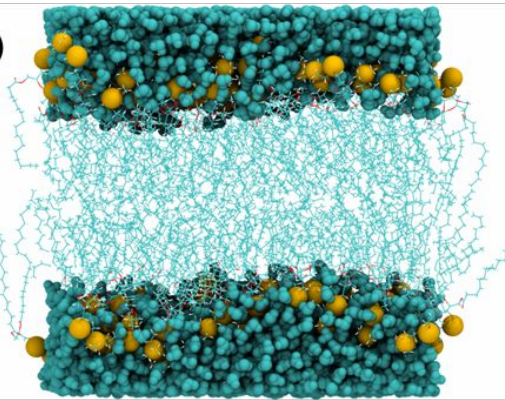

non-oxidized system

(c)

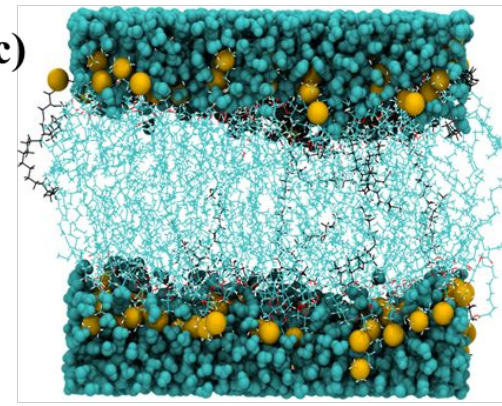

$10 \%$-oxidized system (b)

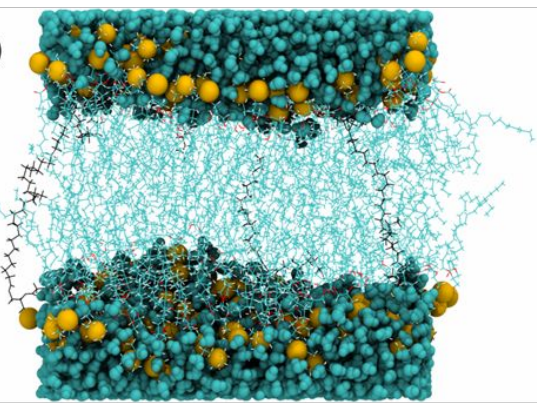

5\%-oxidized system

(d)

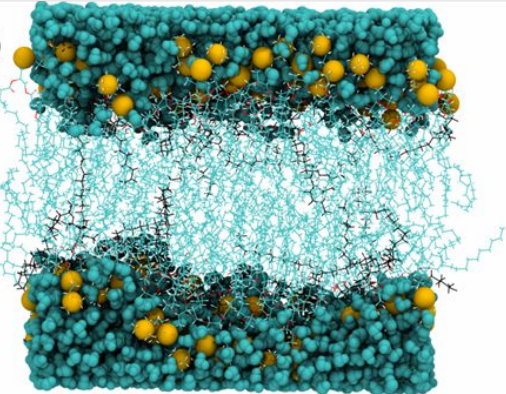

15\%-oxidized system

Figure. S2 Final snapshots of the membrane system with different concentration of oxPLs. (a) 0 , (b) $5 \%$, (c) $10 \%$, (d) $15 \%$. The water molecules are colored in cyan, $\mathrm{N}$ and $\mathrm{P}$ atoms in lipid heads shown in yellow. POPC lipids and alOXs are represented by cyan and black lines

(a)
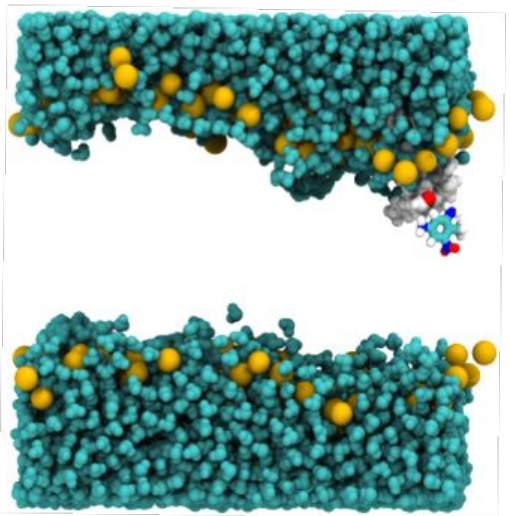

Front view
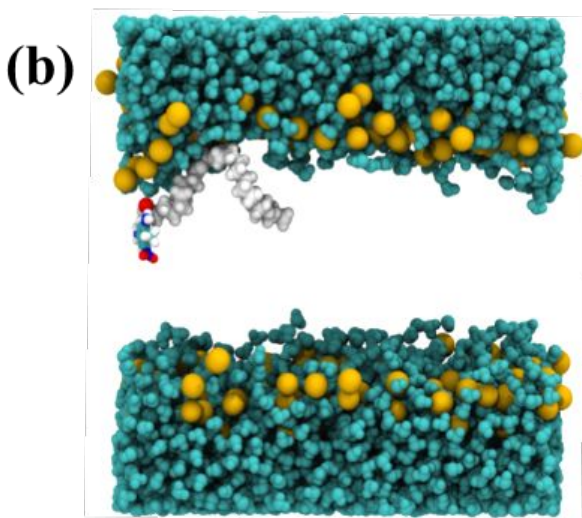

Side view

Figure. S3 The snapshots from the simulation of the close contact of 24DA with the aldehyde groups in the oxidized lipid tails in the $10 \%$-oxidized system, (a) front view, (b) side view. The water molecules are colored in cyan, $\mathrm{N}$ and $\mathrm{P}$ atoms in lipid heads shown in yellow. The tails of selected oxidized lipids are colored in white. The aldehyde groups are presented by red spheres. 

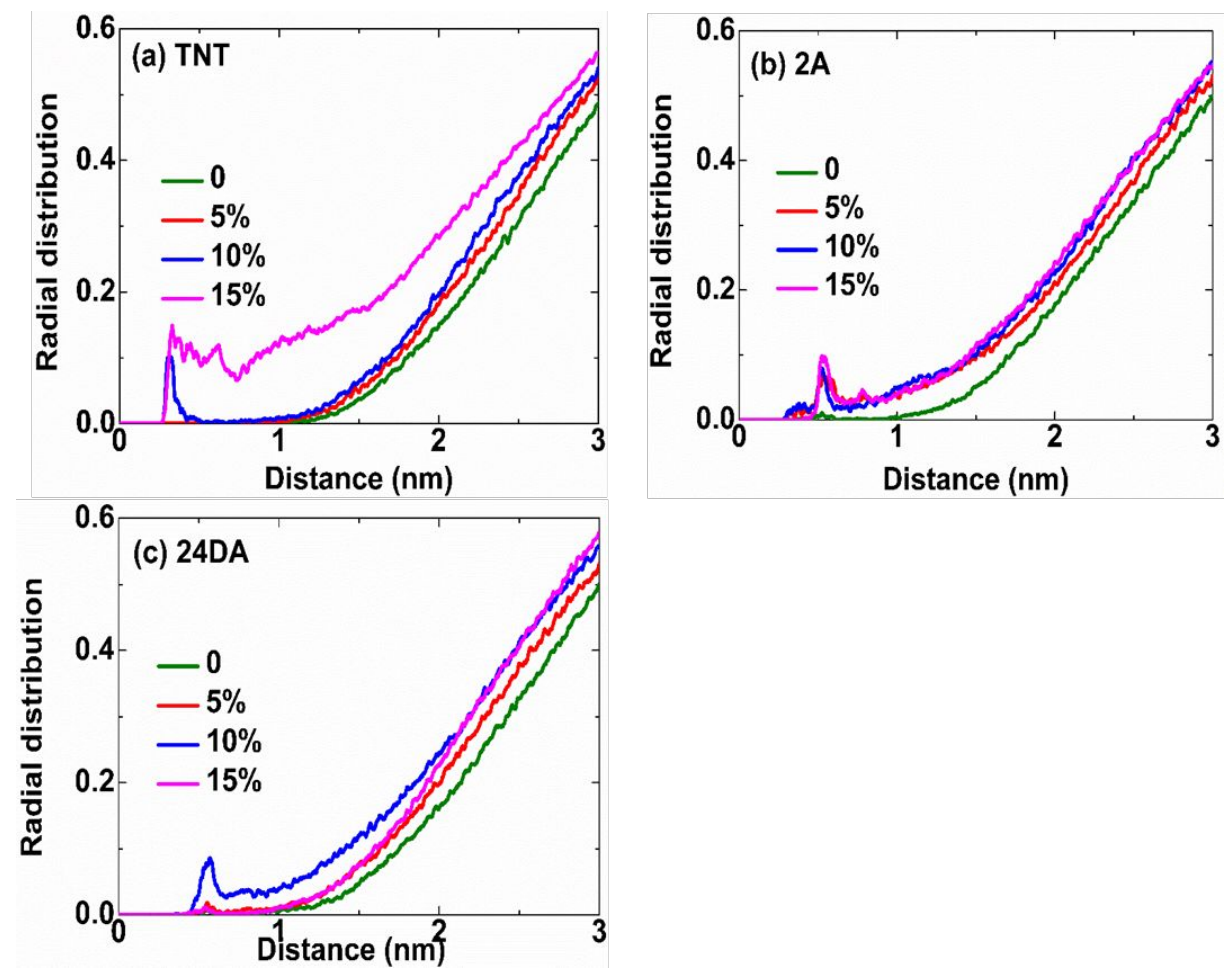

Figure. S4. The radial distribution functions of TNT (a), 2A (b) and 24DA (c) with water molecules at the center of membrane with $0-15 \mathrm{~mol} \%$ of alOXs. 

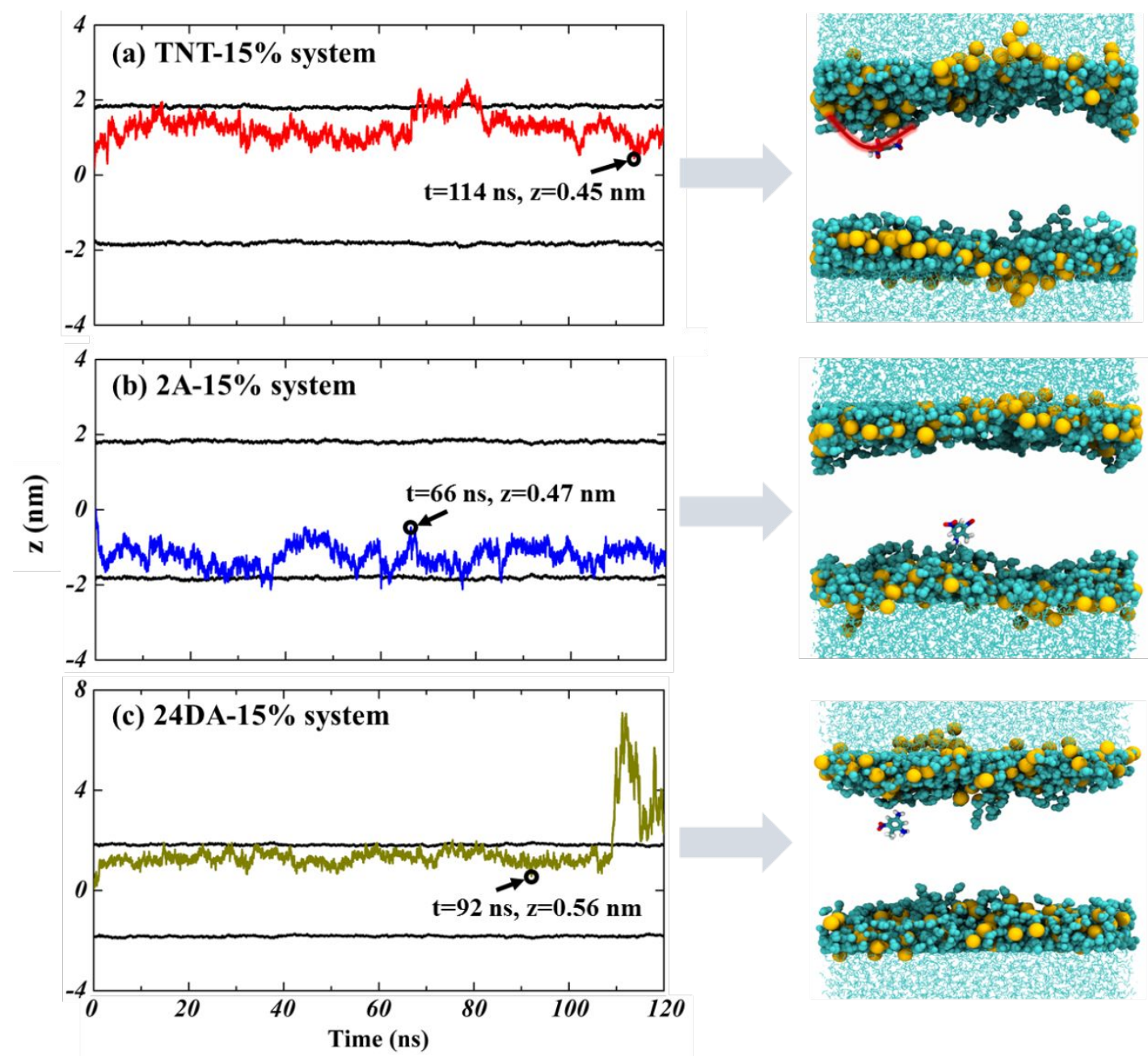

Figure S5. The trajectory of (a) TNT (b) 2A (c) 24DA in the 15\%-oxidized system using the unbiased simulations and the corresponding snapshots in which the solutes remain closest to the bilayer center. The black lines refer to the average position of $\mathrm{P}$ atoms in the different leaflets.

\section{References}

(1) Mayne, C. G.; Saam, J.; Schulten, K.; Tajkhorshid, E.; Gumbart, J. C. Rapid Parameterization of Small Molecules Using the Force Field Toolkit. J. Comput. Chem. 2013, 34, 2757-2770.

(2) Frisch, M. J. E. A. Gaussian 09 Revision D.01. Gaussian Inc. Wallingford CT 2009. 